V.I. TIKHONOV, Dr. of Tech. Sciences, prof., "ONAT", Odessa

\title{
GENERALIZED FOURIER TRANSFORMS BASED ON QUANTUM UNCERTAINTY PRINCIPLE
}

In this paper, basic forms of Fourier analysis considered - harmonic series, integrals and discrete transforms with respect to engineering approach. Quantum uncertainty relation introduced for bound time-frequency metrics in harmonic function presentation. Generalized Fourier transforms determined on the basis of unified summation-integration operator. This extends the scope of harmonic analysis application. Figs.: 2. Refs.: 11 titles.

Keywords: Fourier analysis; quantum uncertainty relation; generalized Fourier transforms; harmonic analysis application.

The problem statement. Many outstanding achievements were obtained on the basis of spectral analysis methods, named after the great French mathematician of the 19th century J. Fourier $(1768-1830)$. In the modern coding theory, data transmission and processing, Fourier analysis is one of the most important mathematical tools, which is based on classical theory of Fourier series and integrals. The classical Fourier series describes a periodic function as a superposition of discrete harmonic functions with a finite or infinite set of frequencies. A lot of physical phenomena can be simulated by periodic processes that adequately modeled by Fourier series. To study nonperiodic functions, Fourier proposed continuous integral transforms. Modern tasks of harmonic analysis widely use discrete spectral transforms. It should be noted, that the classical Fourier analysis operates with idealized models of physical processes, and no method known to clearly divide physical phenomena into classes, which explicitly relevant to harmonic series, integrals or discrete frameworks. This makes difficult comprehensive understanding and practical use of Fourier analysis methods. Therefore, an actual problem rises on further unification of various harmonic analysis forms with particular focus on engineering applications.

Related publications survey. Trigonometric series were used in periodic function analysis before Fourier researches by mathematics d'Alembert, Euler, Bernoulli and Gauss, [1]. In 1755 L. Euler in his "Differential Calculus" wrote a chapter "On the representation of functions by series", [2]. In $1805 \mathrm{~K}$. Gauss, in his unpublished work, presented the method of interpolation of orbital measurements, which can be considered a prototype of modern discrete trigonometric transforms, [3]. In 1807, J. Fourier reported at the Paris Academy his results on the propagation of heat in a solid body, where he firstly described arbitrary functions with the help of trigonometric series. This work was criticized by many famous scientists of that time; in

(C) V.I. Tikhonov, 2017 
1812, J. Fourier received the Academy award, and in 1817 he became a member of the Paris Academy. But only 15 years later, after the death of the academy secretary, J. Fourier published his well-known paper "Analytical theory of heat" (1822), where new method proposed of integral transforms to describe heat transfer processes [4]. The Fourier theory of heat propagation had not been recognized for a long time; later on, this approach, referred to as harmonic Fourier analysis, got various applications (Fourier series and integrals, discrete transforms, fast discrete transforms) [5]; this theory was completed after J. Fourier, when D. Hilbert became an authoritative mathematician of that time (1862-1943). The direct integral Fourier transform maps a given continuous time function whose modulus is integrated on an infinite real time axis, to its spectral image as a function of the continuous frequency, also defined on the infinite real axis, [6]. Integral transforms and Fourier series are effectively used in various fields of physics and geometry, optics, number theory, combinatory, signal processing, probability theory, etc. In fact, in the Fourier series, the periodicity of the process is required, and the Fourier integral considers functions integrated on an infinite time axis, or in an infinite range of continuous frequencies. For these reasons, modern applications of spectral analysis widely apply discrete Fourier transforms (DFT), as well as special algorithmic methods to accelerate the DFT processing, aka fast Fourier transform (FFT), [7].

An increase in the speed of Fourier transforms is an important problem for many technical applications. In this direction, many works contributed. The origins of the DFT and FFT go back to the aforementioned work of Gauss (1805); however, the widely known today algorithm of the FFT was developed after 160 years by IBM engineers (1965), [8]. The terms FFT and DFT are similar in that the FFT is a special algorithm for calculating the DFT, which differs from the direct calculation of the DFT by its determining formulas.

In recent years, works have appeared in the literature in which attempts have been made to construct generalized Fourier transforms, [9 - 11]. In [9] a formula found that relates the Fourier transform of a radial function on $\mathrm{R}_{\mathrm{n}}$ with the Fourier transform of the same function defined on $R_{n+2}$. In [10] a survey in a nutshell given on diverse known forms of Fourier analysis, including the so called short-time Fourier transforms firstly introduced by D. Gabor (1946). In [11] a generalized Fourier transform presented with the use of is the cylindrical Bessel function. We note that in known forms of Fourier analysis, the time and frequency domains commonly are not bound.

Objective. In this paper, we intend to construct generalized Fourier transforms with bound time and frequency domains that include harmonic series, integrals, and discrete Fourier transforms as special cases. 
Methodology aspects of Fourier analysis. In math literature for engineers, the classic Fourier analysis is usually represented by two main sections: "Fourier series" and "Fourier integrals"; an extra item is discrete Fourier transform as a section of numerical methods for solving a wide class of applied problems, [5]. Consider some methodological aspects of Fourier series, integrals and discrete Fourier transforms with respect to engineering applications.

Fourier series. The Fourier series calculus represents a periodic real function $f(t)$ of a real argument $t$ with a repetition period $T$ that satisfies the Dirichlet conditions, as the sum of a trigonometric series. According to the Dirichlet conditions, the $f(t)$ function must be single-valued, piecewisecontinuous and piecewise-monotone (that is, to have a finite number of discontinuities and extrema), and be absolutely integrable (have a finite integral value $\int_{0}^{T}\left|f_{T}(t)\right| d t, \quad[5-7]$. The direct mapping $f(t) \rightarrow\left\{a_{0}, a_{n}, b_{n}\right\}$ of the function $f(t)$ into the set of coefficients of the Fourier series $\left\{a_{0}, a_{n}, b_{n}\right\}, n=1,2, \ldots$, has the form:

$$
\begin{aligned}
& a_{0}=\frac{1}{T} \int_{0}^{T} f(t) d t, \\
& a_{n}=\frac{2}{T} \int_{0}^{T} f(t) \cdot \cos \frac{2 \cdot \pi \cdot n \cdot t}{T} d t, n=1,2, \ldots, \\
& b_{n}=\frac{2}{T} \int_{0}^{T} f(t) \cdot \sin \frac{2 \cdot \pi \cdot n \cdot t}{T} d t, n=1,2, \ldots .
\end{aligned}
$$

The inverse mapping on $\left\{a_{0}, a_{n}, b_{n}\right\} \rightarrow f(t)$ means presentation the function $f(t)$ as Fourier series sum

$$
f(t) \rightarrow a_{0}+\sum_{n=1}^{+\infty}\left(a_{n} \cdot \cos \frac{2 \cdot \pi \cdot n \cdot t}{T}+b_{n} \cdot \sin \frac{2 \cdot \pi \cdot n \cdot t}{T}\right) .
$$

If function $f(t)$ has a finite set of Fourier series terms, than inverse mapping $\left\{a_{0}, a_{n}, b_{n}\right\} \rightarrow f(t)$ in (2) is exhaustive (with some inaccuracy of trigonometric functions calculation). In case of infinite set of Fourier series terms, one can only approximate $f(t)$ by partial sum of trigonometric series. The Fourier series presentation (1) and (2) evolve to more compact complex view in normalized interval $T=2 \cdot \pi$ :

$$
c_{n}=\frac{1}{2 \pi} \int_{0}^{2 \cdot \pi} f(t) \cdot e^{-i \cdot n \cdot t} d t, \quad n=0, \pm 1, \pm 2, \ldots,
$$




$$
f(t) \rightarrow \sum_{n=-\infty}^{+\infty} c_{n} \cdot e^{i \cdot n \cdot t}, \quad t \in[0,2 \pi] .
$$

The complex coefficients $c_{n}$ are related to real numbers $a_{0}, a_{n}, b_{n}$ :

$$
\left\{\begin{array}{lll}
a_{0}=2 \cdot c_{0} ; & a_{n}=c_{+n}+c_{-n} ; & b_{n}=c_{-n}-c_{+n}, \\
c_{0}=\frac{a_{0}}{2} ; & c_{+n}=\frac{a_{n}-i \cdot b_{n}}{2} ; & c_{-n}=\frac{a_{n}+i \cdot b_{n}}{2} .
\end{array}\right.
$$

Integral Fourier transforms. The direct integral Fourier transform is applied to a function $f(t)$ defined on an infinite axis of the independent argument $t \in(-\infty,+\infty)$; as a rule but not necessary, the time axis supposed. The complex Fourier integral is defined with respect to absolutely integrable real or complex function $f(t)$, i.e. such one, that the integral $\int_{-\infty}^{+\infty}|f(t)| d t$ exists and is finite [5-7].

Direct and inverse integral Fourier transforms are often written in symmetrical form:

$$
\left\{\begin{array}{l}
\bar{f}(\omega)=\frac{1}{\sqrt{2 \pi}} \int_{-\infty}^{+\infty} f(t) \cdot e^{-i \cdot \omega \cdot t} d t \\
f(\tau)=\frac{1}{\sqrt{2 \pi}} \int_{-\infty}^{+\infty} \bar{f}(\omega) \cdot e^{i \cdot \omega \cdot t} d \omega .
\end{array}\right.
$$

Understanding Fourier series and integrals relationships. We will compare the relevance of Fourier series and integrals (1-6) with respect to engineering tasks. An actual issue is consideration about Fourier integral transforms as generalized Fourier series. In other words, are Fourier series a particular case of Fourier integrals? Formally, a periodic function that satisfies the Dirichlet conditions is not absolutely integrable in an infinite time interval.

For instance, function $f(t)=\left\{\begin{array}{ll}1, & \text { if } t \in \pm n \cdot[0, \pi] \\ -1, & \text { if } t \in \pm n \cdot[\pi, 2 \pi]\end{array}\right.$ is obviously representable by Fourier series; instead, the integral $\int_{-\infty}^{+\infty}|f(t)| d t=\infty$ does not exist as a finite number. In fact, the requirement of $f(t)$ periodicity for adequate representation by Fourier series can be mitigated, if determined $f(t)$ on a bound normalized interval $t \in[0,2 \pi]$, where $f(t+2 \pi)=f(t)$; herewith, Fourier series calculation remains unchanged, but function $f(t)$ itself turns into absolutely integrable and presentable by Fourier integral [7]. 
It can be shown that the spectrum of such a function will also be discrete, and the inverse integral transformation will become a sum very similar to the sum of the Fourier series. But still, with this approach, the coefficients $c_{n}$ of the Fourier series in (3) do not coincide with the amplitudes $\bar{f}(\omega)$ of the discrete harmonics in (6). This is because of different normalizations in Fourier integral and Fourier series coefficients calculus.

We will represent the direct and inverse transforms of (3) and (4) in symmetric form:

$$
\left\{\begin{array}{l}
c_{n}=\frac{1}{\sqrt{2 \pi}} \int_{0}^{2 \cdot \pi} f(t) \cdot e^{-i \cdot n \cdot t} d t, \quad n=0, \pm 1, \pm 2, \ldots, \\
f(t) \rightarrow \frac{1}{\sqrt{2 \pi}} \sum_{n=-\infty}^{+\infty} c_{n} \cdot e^{i \cdot n \cdot t}, \quad t \in[0,2 \pi] .
\end{array}\right.
$$

Compare (6) and (7) to get the following. If frequency $\omega$ in (6) takes solely integer values $\omega_{n}=n=0, \pm 1, \pm 2, \ldots$, then transforms (7) may consider a special case of integral transforms (6). However, in general, this does not happen. For complete embracing Fourier series by Fourier integral forms, an essential detail needed more, this is a unified way of scaling time $t$ and frequency $\omega$. This aspect discussed onwards in the context of the time-tofrequency quantum uncertainty principle.

Discrete Fourier Transforms. In applications, the discrete form of Fourier transforms (DFT) widely used, as well as special algorithms for accelerated computation of the DFT - the so-called Fast Fourier transforms (FFT), [7-8]. The symmetric form of direct/inverse discrete DFT is:

$$
\left\{\begin{array}{l}
\bar{x}(k)=\sqrt{\frac{1}{N}} \cdot \sum_{n=0}^{N-1} x(n) \cdot \exp \left(-i \cdot \frac{2 \cdot \pi}{N} \cdot k \cdot n\right), \quad k=0,1,2, \ldots, N-1, \\
x(n)=\sqrt{\frac{1}{N}} \cdot \sum_{k=0}^{N-1} x(k) \cdot \exp \left(i \cdot \frac{2 \cdot \pi}{N} \cdot k \cdot n\right), \quad n=0,1,2, \ldots, N-1 .
\end{array}\right.
$$

where $x(n), \bar{x}(k)$ are ordered sets of $N$ real or complex numbers. These sets of numbers can be regarded as the coordinates of vectors in some complex space; so, for simplicity, we will designate both $x(n)$ and $\bar{x}(k)$ as vectors $x$ and $\bar{x}$, if no ambiguity seemed. The discrete Fourier transforms can also be written in terms of matrix or tensor operations for convenient program coding.

Let complex kernels of sum operators (8) be complex conjugate unitary matrices $F$ and $F^{*}$ with the property $F^{*} \cdot F=F \cdot F^{*}=I$, where $I$ is the diagonal unit matrix: 


$$
\left\{\begin{array}{l}
F^{*}=F^{*}(n, k)=\sqrt{\frac{1}{N}} \cdot \exp \left(-i \cdot \frac{2 \cdot \pi}{N} \cdot k \cdot n\right), \\
F=F(k, n)=\sqrt{\frac{1}{N}} \cdot \exp \left(i \cdot \frac{2 \cdot \pi}{N} \cdot n \cdot k\right) .
\end{array}\right.
$$

With notation introduced above, the DFT pair becomes:

$$
\left\{\begin{array}{l}
\bar{x}=x \cdot F^{*}, \\
x=\bar{x} \cdot F .
\end{array}\right.
$$

Substitute $\hat{x}$ from the first into the second transform (10), we obtain

$$
x=\bar{x} \cdot F=\left(x \cdot F^{*}\right) \cdot F=x \cdot\left(F^{*} \cdot F\right)=x \cdot I=x .
$$

The unitarity of discrete Fourier transform matrix. The unitarity of the discrete Fourier transform matrix is the key feature to validate the one-toone mapping property of $(x=\bar{x} \cdot F) \leftrightarrow\left(\bar{x}=x \cdot F^{*}\right)$. Unlike rather abstract reasoning for convergence Fourier series sum and inverse integral Fourier transform $[6,7]$, the unitarity of the discrete transformation matrix is simply derived by elementary geometric progression and primitive complex unity root [9].

Let a geometric progression given as a numerical sequence $\left\{a_{k}\right\}$ with a real or complex denominator $q \neq 1$ :

$$
\left\{a_{k}\right\}=a_{0}, a_{0} \cdot q, a_{0} \cdot q^{2}, \ldots=\left\{a_{0} \cdot q^{k}\right\}, k=0,1,2, \ldots .
$$

The partial sum of a segment of this sequence is calculated by the following formula, [5]:

$$
\sum_{k=L}^{K}\left\{a_{k}\right\}=\frac{a_{L}-a_{K} \cdot q}{1-q}, \quad L<K .
$$

In particular, the initial sum of the progression terms from 0 to $N$ is

$$
\sum_{k=0}^{N}\left\{a_{k}\right\}=\frac{a_{0}-a_{N} \cdot q}{1-q} .
$$

Let consider a special case of a geometric progression - the so-called cyclic progression, in which the initial and final elements of a periodic segment match, i.e. $a_{L}=a_{K}, L<K$. In this case, the sum of the cyclic segment terms is simplified 


$$
\sum_{k=L}^{K}\left\{a_{k}\right\}=\frac{a_{L}-a_{L} \cdot q}{1-q}=a_{L} \cdot \frac{1-q}{1-q}=a_{L} .
$$

In particular, if $a_{0}=a_{K}=1$, then (15) evolves to $\sum_{k=0}^{K}\left\{a_{k}\right\}=1$. This will be used in consideration the primitive complex unity root $1_{C}$ defined as [9]:

$$
1_{C}:=\exp (i \cdot 2 \cdot \pi), \quad i:=\sqrt{-1} .
$$

The following property of the primitive root $1_{C}$ holds: if some number $\mu$ is an integer, then the expression $\left(1_{C}\right)^{\mu}$ equals 1 ; if $\mu$ not integer, then $\left(1_{C}\right)^{\mu} \neq 1:$ integer

$$
\left(1_{C}\right)^{\mu}\left\{\begin{array}{l}
=1, \text { if } \mu \text { is integer number, } \\
\neq 1, \text { if } \mu \text { is not integer number. }
\end{array}\right.
$$

It is not hard to find property (17) using the well-known Euler formula $\exp (i \cdot \varphi)=\cos \varphi+i \cdot \sin \varphi$, taking into account that $\cos 2 \pi=1$, and $\sin 2 \pi=0$. Now, consider the following sequence

$$
\left\{\exp \left(i \cdot \frac{2 \cdot \pi \cdot \mu}{N}\right)^{k}\right\}=\left\{\left[\left(1_{C}\right)^{\frac{\mu}{N}}\right]^{k}\right\},|\mu|<N ; k=0,1,2, \ldots, N .
$$

According to the primitive root $1_{C}$ property (17), along with $|\mu|<N$, the $q=\left(1_{C}\right)^{\frac{\mu}{N}}$ can be denominator of the following geometric progression

$$
\left\{\left[\left(1_{C}\right)^{\frac{\mu}{N}}\right]^{k}\right\}=\left\{q^{k}\right\},|\mu|<N ; k=0,1,2, \ldots, N, \ldots .
$$

Obviously, the initial and final terms of sequence (19) have the same values: $a_{0}=q^{0}=1 ; a_{N}=q^{N}=\left[\left(1_{C}\right)^{\frac{\mu}{N}}\right]^{N}=\left(1_{C}\right)^{\frac{\mu}{N} N}=\left(1_{C}\right)^{\mu}=1$, as $\mu$ is an integer number. The partial sum of (19) is

$$
\sum_{k=0}^{N}\left[\left(1_{C}\right)^{\frac{\mu}{N}}\right]^{k}=\sum_{k=0}^{N} q^{k}=1
$$

Since the last term of the series (19) is 1 , it follows from (20) that partial sum of (19) omitting the last term with number $N$, is zero: 


$$
\sum_{k=0}^{N-1}\left[\left(1_{C}\right)^{\frac{\mu}{N}}\right]^{k}=\sum_{k=0}^{N} q^{k}-1=0
$$

The formula (21) is the principal key to justify the unitarity of the DFT matrix. Consider the product of DFT (8) substituting $n$ by $m$ in $F$ matrix defined in (9):

$$
F^{*} \cdot F=\frac{1}{N} \cdot \sum_{k=0}^{N-1} F^{*}(n, k) \cdot F(k, m)=\frac{1}{N} \cdot \sum_{k=0}^{N-1} \exp \left(i \cdot 2 \cdot \pi \cdot \frac{(n-m)}{N} \cdot k\right) .
$$

Denote $\mu=n-m$. Since $n<N, m<N$, it is $|\mu|=|n-m|<N$ and $\frac{\mu}{N} \neq 1$; therefore, $\exp \left(i \cdot 2 \cdot \pi \frac{(n-m)}{N} \cdot k\right)=\left[\exp \left(i \cdot 2 \cdot \pi \frac{\mu}{N}\right)\right]^{k}=\left[\left(1_{C}\right)^{\frac{\mu}{N}}\right]^{k}$. Compare the last expression with (21), we can see that $F^{*} \cdot F=1$ for $n=m$, and $F^{*} \cdot F=0$ for $n \neq m$, i.e. $F, F^{*}$ are unitary matrices. Hence it is also easy to show that $F \cdot F^{*}=\left(F^{*} \cdot F\right)^{*}=I^{*}=I$.

Generalized Fourier Transforms. It was shown above that integral Fourier transforms (6) look like embracing the Fourier series (3); however, the non-correspondence of time/frequency scaling in Fourier series (3) and integrals (6) hinders complete unification of these two forms of harmonic analysis. These issues are discussed below in more details.

Consider relationship between Fourier series and DFT. Suppose vector $x=x(n)$ as function $\bar{f}(t)$ quantization product, assuming that $\bar{f}(t)$ determined on a bounded half-open interval $t \in[0, T)$ and satisfied the Dirichlet conditions (in order to be representable by Fourier series). Despite function $\bar{f}(t)$ is not periodic in our case, the time interval $[0, T)$ can be interpreted as a part of an extended time interval $0 \leq t<\infty$ [7]

$$
\bigcup\left\langle[0, T)_{1},[0, T)_{2},[0, T)_{3}, \ldots\right\rangle=[0,3 \cdot T), \ldots \rightarrow\langle 0 \leq t<\infty\rangle .
$$

Now, the closure of a half-open interval $[0, T)$ by a point $t=T$ together with its neighborhood, along with function definition in the neighborhood of $T$ under condition $f(0)=f(T)$, allows function $\bar{f}(t)$ in a half-open interval $t \in[0, T)$ considered as one period of a periodic function $f(t)$ determined on the interval $0 \leq t<\infty$. Function $f(t)$ defined on a closed interval $[0, T]$ of the real axis $t \in R$ where $f(0)=f(T)$, we denote as quasi-periodic function with a half-open repetition period $t \in[0, T)$ that is closed on the left and is open to the right. However, integration $f(t)$ on entire 
closed interval $[0, T]$ in (1) is not absolutely accurate to our mind, as point $t=T$ does not belong to the period of function $f(t)$. This aspect seems to be the core issue in unification diverse forms of harmonic analysis. Fig. 1 shows vector $x=x(n)$ as quantified function $f(t)$ on interval $t \in[0, T]$ partitioned in 8 quanta $\Delta t=\frac{T}{8}$. Eight discrete function samples are taken at the beginning of each $\Delta t$ quantum. The last point $n=9$ of the interval does not belong to the period of function repetition and does not carry additional information, since $x(9) \equiv x(0)$. To overcome the issue of the wrong extra point within the closed integration interval $t \in[0, T]$ of a periodic function $f(t)$, we propose to change the commonly used open time axis $-\infty<t<+\infty$ for a closed loop-time, or topological time-circle $C$ with normalized length $\sqrt{2 \cdot \pi}$. Herewith, two marginal points of closed linear interval $t \in[0, T]$ will merge into one joint point $t=0=\sqrt{2 \cdot \pi}$. Next, we postulate the quantum uncertainty relationship (QUR) to bound frequency and time arguments along with known Heisenberg uncertainty principle in quantum physics [10]

$$
\Delta t \cdot \Delta \omega=\frac{2 \cdot \pi}{N} .
$$

Define symmetric normalized form of QUR [11]

$$
\Delta t=\Delta \omega=\sqrt{\frac{2 \cdot \pi}{N}} .
$$

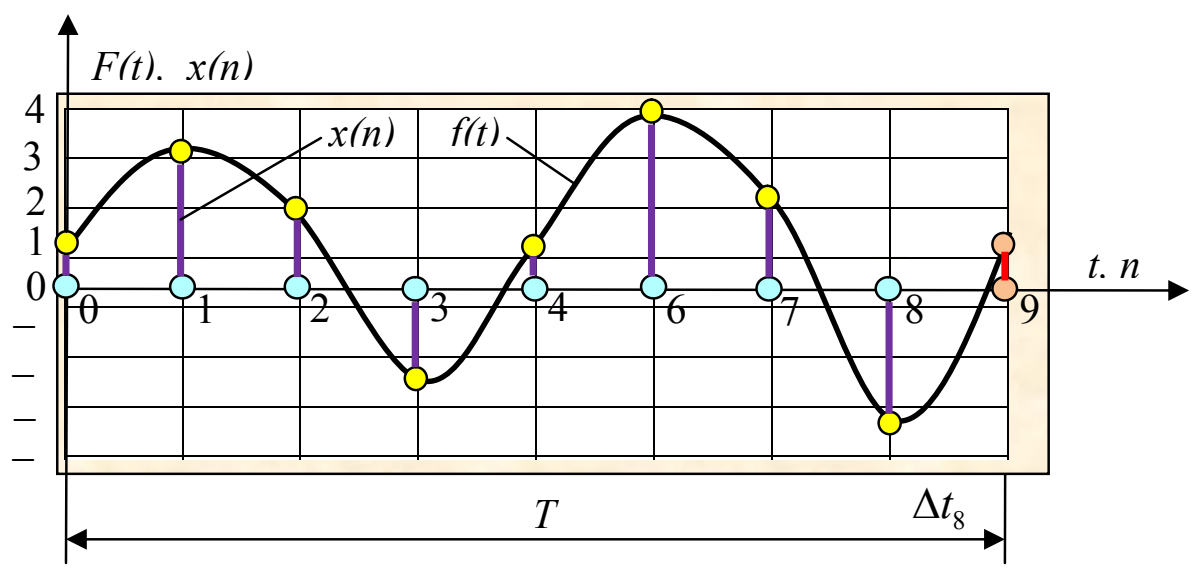

Fig. 1. Vector of function quantization in time 
In this case, time and frequency arguments $t, \omega \in C$ vary in normalized time-circle interval $C$. Here, DFTs (8) evolve to integral sums along unified contour $C[5,6]$ :

$$
\left\{\begin{array}{l}
\bar{x}(\omega)=\frac{1}{\sqrt{2 \cdot \pi}} \cdot \sum_{t \in C} x(t) \cdot \exp (-i \cdot \omega \cdot t) \cdot \Delta t \\
x(t)=\frac{1}{\sqrt{2 \cdot \pi}} \cdot \sum_{\omega \in C} \bar{x}(\omega) \cdot \exp (i \cdot t \cdot \omega) \cdot \Delta \omega .
\end{array}\right.
$$

Substitute $\bar{x}(\omega)$ in the second equation of (26) for its form in the first equation (26), changing argument $t$ for $\tau$ in the second equation (26), we obtain the following identity:

$$
\begin{gathered}
x(\tau)=\frac{\Delta t \cdot \Delta \omega}{2 \cdot \pi} \cdot \sum_{t \in C} x(t) \cdot \sum_{\omega \in C} \exp [i \cdot(\tau-t) \cdot \omega]= \\
=\sum_{t \in C} x(t) \cdot I(\tau, t)=x(\tau) .
\end{gathered}
$$

Introduce "unified summation-integration operator" (USO) on a circle interval, which is scaled to the standard interval $C$ of $\sqrt{2 \cdot \pi}$ length; it is similar to common contour integral, so denote USO as $\oint_{t} f($ ) in time domain or $\int_{\omega} f($ ) in frequency domain. For example,

$$
\oint_{t} f()=\lim _{\Delta t \rightarrow 0} \frac{1}{\sqrt{2 \cdot \pi}} \cdot \sum_{t \in C} f() \cdot \Delta t .
$$

Depending on the context, we understand $\int f($ ) as integral sum or as a contour integral where $N \rightarrow \infty, \Delta_{N \rightarrow \infty}=\frac{2 \cdot \pi}{N} \rightarrow 0$. Rewrite omitting $d t$ or $d \omega$ as default given symbols:

$$
\left\{\begin{array}{l}
\bar{x}(\omega)=\oint_{t} x(t) \cdot \exp (-i \cdot \omega \cdot t), \\
x(t)=\oint_{\omega} \bar{x}(\omega) \cdot \exp (+i \cdot t \cdot \omega) .
\end{array}\right.
$$

A pair of equations (29) we define as generalized Fourier transforms (GFT). Due to (27), it is clear that GFTs provide one-to-one mapping of the original function $x(t)$ into its spectral image $\bar{x}(\omega): x(\tau) \leftrightarrow \bar{x}(\omega)$ for any finite or infinite number of intervals of partitioning the argument, starting from the value $N=2$ (solely inaccuracy may occur in approaching trigonometric 
functions). The GFT introduced above may have two marginal interpretations - discrete form (if $N>1$ is finite digital number) and continuous form (if $N \rightarrow \infty, \Delta t, \Delta \omega \rightarrow 0$ ), Fig. 2. In discrete interpretation form, the GPF (29) is equivalent to DFT with special scaling in (8). In continuum interpretation form, we obtain unified transforms which include Fourier series and integrals as particular cases among many other intermediate forms.

Besides, depending on specific spectrum distribution $|\bar{x}(k)|^{2}$, two extreme typical cases observed (both in the discrete and continuum forms):

1) Prototype of Fourier series (spectrum $|\bar{x}(k)|^{2}$ concentrated at points of a multiple frequency); when $N \rightarrow \infty, \Delta t \rightarrow 0$, we obtain Fourier series;

2) Prototype of Fourier integral (spectrum $|\bar{x}(k)|^{2}$ distributed fairly evenly); when $N \rightarrow \infty, \Delta t \rightarrow 0$, we obtain Fourier integral.

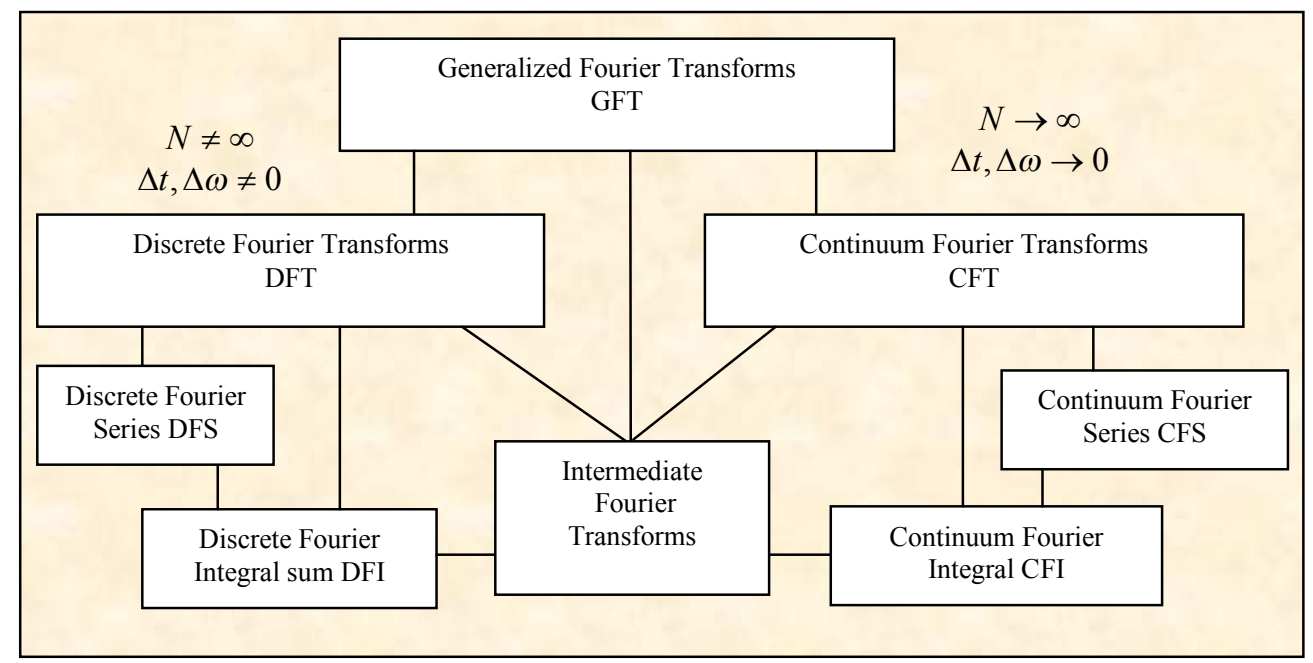

Fig. 2. Interpretation diversity of generalized Fourier transforms

Based on generalized Fourier transforms (GFT), it becomes rather conditional in separation the classes of functions representable by Fourier series or integrals, since many intermediate forms can be observed by GFT. Similarly, no sense to categorize discrete and continuum Fourier transforms, as in limit $N \rightarrow \infty$, the GFT embraces both Fourier series and integrals among the other transitional forms. Given approach seems reasonable for practical applications and theoretical study by engineers.

Conclusion. An adequate utilization of theoretical methods is a nontrivial task in applied researches. The classic approach provides at least three widely known forms of signal representation by trigonometric functions (Fourier series, Fourier integrals and discrete Fourier transforms), as well as variety of application algorithms; this raises methodological issues for 
development engineers caused by the lack of clear problem identification for relevant methods, as well as the absence of exact formalized relationships between the three forms of Fourier analysis. In this work, unification of three popular forms of Fourier harmonic analysis introduced based on fundamental physical principle of quantum uncertainty; this principle formulated as quantum uncertainty relation between the time and frequency arguments. Similar to classic Riemann integral, presented a unified summation-integration operator (USO) with symmetrically scaled variables of time and frequency; due to the USO, symmetric forms of generalized direct/inverse Fourier transforms constructed. These observations provide a unified method of harmonic Fourier analysis both in theoretical and empirical planes.

\section{References:}

1. Anand. A.V. (2010), A brief study of discrete and fast Fourier transforms (1. History and introduction), available at: http://www.math.uchicago.edu/ may/VIGRE/VIGRE2010/ REUPapers/Anand.pdf.

2. "E212 - Institutiones calculi differentialis cum eius usu in analysi finitorum ac doctrina serierum (Foundations of Differential Calculus, with Applications to Finite Analysis and Series)", available at: http://eulerarchive.maa.org//pages/E212.html.

3. Heideman M., Johnson D., and Burrus C. (1985), "Gauss and the history of the fast Fourier transform", Archive for History of Exact Sciences, 1985, 34 (3), pp. 265-277.

4. Fourier J. (1988), Théorie analytique de la chaleur, A. Paris, Firmin Didot Père et Fils, 1822, 639 p., available at: https://www.irphe.fr/ clanet/otherpaperfile/articles/Fourier/ N0029061_PDF_1_676.pdf.

5. Korn G., and $\overline{\text { K}}$ orn T. (1968), "Mathematical handbook for scientists and engineers", McGraw-Hill Book Co., N.Y., 1097 p.

6. Buhler T., and Salamon D.A. (2017), "Functional Analysis", ETH Zurich, available at: https://people.math.ethz.ch/ salamon/PREPRINTS/funcana.pdf.

7. Osgood B. "Lecture Notes for EE 261. The Fourier Transform and its Applications", Electrical Engineering Department, Stanford University, 422 p., available at: https://see.stanford.edu/materials/lsoftaee261/book-fall-07.pdf.

8. Cooley J., and Tukey J. (1965), "An algorithm for the machine calculation of complex Fourier series", Math. Comput., available at: http://garfield.library.upenn.edu/ classics1993/A1993MJ84400001.pdf.

9. Grafacos L., and Teschl G. (2013), "On Fourier transforms of radial functions and distributions", available at: https://arxiv.org/pdf/1112.5469.pdf.

10. Muller M. (2015), "The Fourier Transform in a Nutshell", available at: https://www.researchgate.net/publication/290440858

The_Fourier_Transform_in_a_Nutshell.

11. Yao J., Williams C.L., Hussain F., and Kouri D.J. (2017), "Generalized Fourier transform method for nonlinear anomalous diffusion equation", available at: https://arxiv.org/pdf/1701.03488.pdf.

12. McClellan J., and Rader C., (1979), "Number theory in digital signal processing", Prentice-Hall, Inc., Englewood Cliffs, N.J., 288 p.

13. Hizenberg W., (1949), "The physical principles of the quantum theory", available at: https://books.google.com.ua/books?id=T9zCAgAAQBAJ\&printsec=frontcover\&dq=Heisenbe rg+principl.

14. Tichonov V.I., (1981), "Umkehraufgaben und Datenbevertung", Berichte der MathematischStatistischen Sektion im Forschungszentrum Graz, Bericht Nr. 172, 106 p. 
The article is presented by doctor of technical sciences, professor, the head of "Radio and Television" depart. of the "Institute of Radio, Television and Information Security" of "O.S. Popov ONAT", Honored Worker of Science and Technology of Ukraine Gofiyzen O.V.

Tikhonov Victor

Doctor of technical science, associate professor

Laureate of the Ukraine State Prize for Science and Technology,

Professor of Communication Networks department

O.S. Popov Odessa National Academy of Telecommunications

1 Kuznechna St., Odessa 65029, Ukraine

Tel.: 067-752-13-90

E-mail: victor.tykhonov@onat.edu.ua 
УДК 519.6

Узагальнені перетворення Фур'є на основі принципу квантової невизначеності. / Тіхонов В.І. // Вісник НТУ "ХПІ". Серія: Інформатика та моделювання. - Харків: НТУ "ХПІ". - 2017. - № 21 (1243). - С. 103 - 116.

У статті розглядається питання гармонійного аналізу функцій, в тому числі рядів Фур'є, інтегралів і дискретних перетворень 3 точки зору інженерних застосувань. Введено квантове співвідношення невизначеностей для пов'язаних метрик часу частоти у частотному представленні функції часу. Визначені узагальнені перетворення Фур'є на основі уніфікованого оператора сумування-інтегрування. Іл.: 2. Бібліогр.: 11 назв.

Ключові слова: гармонійний аналіз функцій; співвідношення квантової невизначеності; узагальнені перетворення Фур'є.

\section{УДК 519.6}

Обобщенные преобразования Фурье на основе принципа квантовой неопределенности / Тихонов В.И. // Вестник НТУ "ХПИ". Серия: Информатика и моделирование. - Харьков: НТУ "ХПИ". - 2017. - № 21 (1243). - С. 103 - 116.

В статье рассматриваются вопросы гармонического анализа функций, в том числе ряды Фурье, интегралы и дискретные преобразования с точки зрения инженерных приложений. Введено отношение квантовой неопределенности для связанных частотновременных метрик в частотной представлении функции времени. Определены обобщенные преобразования Фурье на основе унифицированного оператора суммирования-интегрирования. Ил.: 2. Библиогр.: 11 назв.

Ключевые слова: гармонический анализ функций; отношение квантовой неопределенности; обобщенные преобразования Фурье.

\section{UDC 519.6}

Generalized Fourier transforms based on quantum uncertainty principle / Tikhonov V.I. // Herald of the National Technical University "KhPI". Subject issue: Information Science and Modelling. - Kharkov: NTU "KhPI". - 2017. - №. 21 (1243). - P. 103 116.

In this paper, basic forms of Fourier analysis considered - harmonic series, integrals and discrete transforms with respect to engineering approach. Quantum uncertainty relation introduced for bound time-frequency metrics in harmonic function presentation. Generalized Fourier transforms determined on the basis of unified summation-integration operator. This extends the scope of harmonic analysis application. Figs.: 2. Refs.: 11 titles.

Keywords: Fourier analysis; quantum uncertainty relation; generalized Fourier transforms; harmonic analysis application. 